\title{
Two LCOS full color projector with efficient LED illumination engine
}

\author{
Hüseyin Murat**a ${ }^{a y k u t ~ A v c i ~}{ }^{\mathrm{a}}$, Roel Beernaert ${ }^{\mathrm{a}}$, Kristof Dhaenens ${ }^{\mathrm{b}}$, Herbert De Smet ${ }^{\mathrm{a}, \mathrm{b}}$, \\ Lawrence Bogaert ${ }^{\mathrm{c}}$, Bart Van Giel ${ }^{\mathrm{c}}$, Youri Meuret ${ }^{\mathrm{c}}$ \\ ${ }^{a}$ Ghent University, Department of Electronics and Information Systems, \\ TFCG Microsystems group \\ ${ }^{\mathrm{b}}$ Interuniversity MicroElectronics Center, Information Technology Division \\ Technologiepark 914A, B-9052 Ghent, Belgium \\ ${ }^{c}$ Department of Applied Physics and Photonics, Vrije Universiteit Brussel \\ Pleinlaan 2, B-1050 Brussels, Belgium
}

\begin{abstract}
LED-based projectors have numerous advantages compared to traditional projectors. They are more compact, exhibit a larger color gamut and a longer lifetime, the supply voltage is lower and they can operate on batteries. However their light output is limited by the low optical power per étendue of the LEDs, although this is also improving constantly. With an efficient illumination engine design we can build an LED projector with a moderate light output and with superior properties. We present a compact LED projector with two 'Liquid Crystal On Silicon' (LCOS) panels. One of these panels is alternately modulating red and blue information while the other permanently modulates the green information to achieve the D65 white point. This architecture is first simulated using ray-tracing software. After testing the several parts of this architecture experimentally, we have built a demonstrator setup. Our demonstrator produces a moderate light output $(37.3 \mathrm{~lm})$ on screen with a sufficient contrast ratio and a very good uniformity, within a system étendue lower than $20 \mathrm{~mm}^{2} \mathrm{sr}$. In spite of semi color sequential working, the color breakup and crosstalk are negligible.
\end{abstract}

Keywords: LED, QXGA LCOS, projector, gradually tapered light pipe

\section{INTRODUCTION}

The market for digital projectors is one of the fastest growing markets. There are many projection applications such as 'business projectors', 'home theater projectors', 'rear projection television', 'handheld projectors', '3D Displays', etc. Projectors are not only used in professional environments but are progressively entering the consumer market. The challenge is to design high quality, durable, low cost and compact projectors. There is much research in this domain. LED-based compact projectors are one of the hot topics. The light flux of LEDs has improved consistently since their invention. This progress makes it recently interesting to use these devices as light sources for projection applications. Compared with traditional light sources, for example the mercury arc discharge lamps, LEDs have numerous advantages ${ }^{1,2}$. They are small and robust, have a longer lifetime, exhibit a relatively narrow spectral emission band, resulting in a larger color gamut, and are directly available in R, G, B colors; the supply voltage is lower, they can switch on and off very rapidly, have a high dimming ratio that can be used to improve the contrast, they emit no UV, do not contain dangerous mercury etc. One disadvantage is the significantly lower luminance (optical power per unit of étendue $^{3}$ ). Because of this and the étendue limitation of the projector (size limitation of the light valve, f-number limitation of the projection system), the light output of a LED projector is currently limited, but Haitz ${ }^{4,14}$ law predicts a steady grow of the lumen output per LED package in the future 5 . Despite this shortcoming, LEDs are still very interesting for moderate light output applications because of their superior properties, but they should be designed efficiently. Using three primary color light sources makes a compact projector design possible. In this paper we present 
a LED projector with two "liquid crystal on silicon" (LCOS) light valves and four LEDs (red, 2x green, blue). A detailed description of the architecture is given in the following section.

\section{DESIGN OF LED-BASED TWO LCOS PROJECTOR}

Because of the different nature of LED light sources, the architecture of the classic projector must be adapted. The principal changes will be in the illumination part of the projector. As light valves, we have chosen LCOS panels. Alternatives could be high-temperature poly-silicon (HTPS) LCDs or digital micro-mirror devices (DMD ${ }^{\mathrm{TM}}$ ). The added value of LCOS panels is the very high resolution capability, their high fill factor, the analogous modulating character, and their availability and relatively low cost. We designed, simulated and optimized the projector setup using the optical design software ZEMAX. Further we built a demonstrator setup and characterized it experimentally.

\subsection{The projector architecture}

Before we discuss the architecture, we describe the projector parameters and the assumptions. We assumed an LCOS light valve with a diagonal of 0.82 " and an aspect ratio of $4: 3$, which means the dimensions $(\mathrm{WxH})$ are $16.66 \mathrm{~mm} \mathrm{x}$ $12.50 \mathrm{~mm}$. The system f-number is 2.88 which corresponds with rays of maximum 10 degrees top angle. The f-number is limited not only by lens cost considerations, but also by contrast requirements. With these assumptions we can calculate the system étendue, i.e. $19.73 \mathrm{~mm}^{2} \mathrm{sr}$. We chose the illumination area somewhat larger than the light valve to have a certain overfill, which enables us to illuminate the micro display uniformly including the corners. The illumination area is $17.6 \mathrm{~mm} * 13.2 \mathrm{~mm}$, so we have almost $12 \%$ overfill. The aspect ratio is again $4: 3$. The rescaled system étendue is $22.01 \mathrm{~mm}^{2} \mathrm{sr}$.

In this work we used OSTAR Projection LEDs from OSRAM ${ }^{6}$. The package type we used has 6 dies (LE X H3A) but we drove only 4 dies and thus a total surface of $2.1 \mathrm{~mm} \times 2.1 \mathrm{~mm}$. They have a lambertian radiation pattern and are truly surface emitters. They have no encapsulation dome and are emitting directly into the air. So we can couple them optically to the collecting light pipes. The source étendue will be $31.88 \mathrm{~mm}^{2} \mathrm{sr}$ or $30.67 \mathrm{~mm}^{2} \mathrm{sr}$ depending on the used material for the light pipe (BK7 in the simulations and Plexiglas in the experimental setup). This is roughly $50 \%$ more than the system étendue, which means that even in the ideal case, not all the rays will pass through the system.

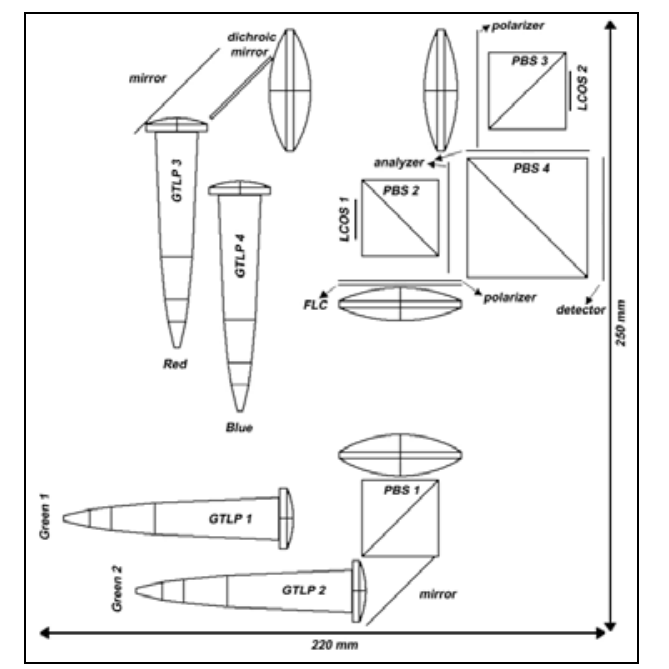

Figure 1: Schematic architecture of the LED projector

The architecture of the LED projector is given in figure 1. It is a two-light-valve system where one LCOS is continuously reserved for the green channel and the other for the red and blue channels alternately. These two paths are totally separated so that crosstalk between the channels is avoided. The recombining at the end is done by a large 
'polarizing beam splitter' (PBS). The PBS and the lenses are antireflection (AR) coated. All these components' losses are taken into account. In the illumination part, the main component is the "Gradually Tapered Light Pipe" (GTLP). In the following section we will describe in detail the principal parts of the projector.

\subsection{LED illumination engine with GTLPs}

LEDs have a totally different shape, radiation pattern, driving requirement, etc. than classic light sources. Therefore the illumination engine, which determines the brightness and the uniformity of the system, must be redesigned. We developed an illumination system based on custom made 'Gradually Tapered Light Pipes' (GTLP) $)^{7}$. These are components that act as collector and integrator and at the same time transform the lambertian radiation pattern into a pattern with the desired acceptance angle. The entrance surface is $2.2 \mathrm{~mm}$ x $2.2 \mathrm{~mm}$ and the exit window $17.6 \mathrm{~mm} \times$ $13.2 \mathrm{~mm}$ (ratio $4: 3$ ). The cross section is gradually enlarging along the length $(90 \mathrm{~mm})$ of the pipe. The LED die $(2.1 \mathrm{~mm}$ $\mathrm{x} 2.1 \mathrm{~mm}$ ) is optically coupled to the GTLP by using index matching gel. The collected rays are propagating through the massive body (BK7 glass or Plexiglas) of the pipe by the principal of total internal reflection (TIR). The number of reflections in the pipe is a measure for the uniformity. At the end of the pipe, the spatial and the angular distributions of the light spot are adapted to the requirements, which means a uniformly illuminated rectangle (4:3 ratio and the size of LCOS including overfill) and an adapted angular distribution within the premised f-number (2.88). The light beam at the exit window is telecentric and could directly illuminate the light valve in case of transmissive LCD panels. Telecentric illumination means that each point of the rectangular beam has the same angular distribution. That is very important for the screen uniformity of the projector system. Otherwise, because of the angle-dependent behavior of some optical components (PBS, X cube, etc.), the uniformity of the imaged beam will degrade. In our case we still require a one-toone image that is realized by a telecentric three-lens system. The exit window of the pipe is placed in the object space and the LCOS panel in the image space of the system. The lenses are standard, commercial lenses. The brightness, uniformity and the efficiency of the GTLP will be presented later.

\subsection{Green channel}

Unlike the systems based on arc discharge lamps, green is the limiting color ${ }^{2}$ in today's LED-based projectors. To have a color-balanced LED projector, the green color channel should have an increased light input compared to the other color channels. In practice, we require roughly two green LEDs for one blue and one red LED. Therefore we designed a two LCOS system where one panel is continuously illuminated by green LEDs and the other sequentially illuminated by the blue and the red LED. In this section the green channel will be explained.

LCOS 1 could be continuously illuminated by one green LED, but we opted for another solution that is shown in figure 2. Instead of one LED continuously driven at nominal current we used the pulsing capability of LEDs and we pulsed two green LEDs alternately at a higher current (twice the nominal current). The brightness of each pulsed LED is higher than in the nominal driving case but only during $50 \%$ of the time. Now the increased brightness can be added up in time and will generate a higher average brightness. By applying this technique ${ }^{1}$ we achieved a substantial net gain of $36 \%$ with Luxeon LEDs. This gain is strongly depending on the type of LEDs and PBS. With the components (OSTAR ${ }^{6}$ and $\mathrm{HELF}^{\mathrm{TM} 8} \mathrm{PBS}$ ) that we used in our setup, this gain will only be $15 \%$. 


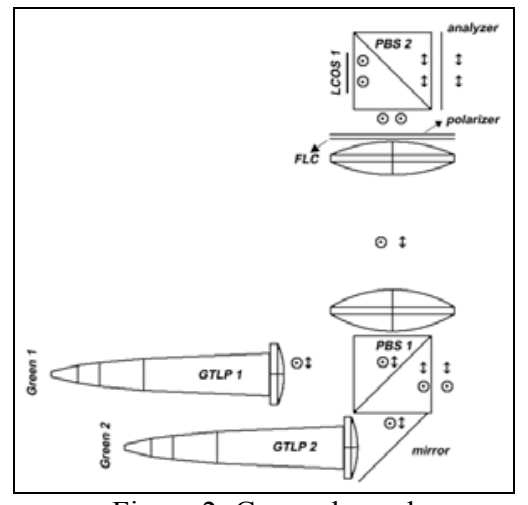

Figure 2: Green channel

First the LED indicated as "green1" is pulsed. As shown in figure 2, PBS1 reflects the s-polarization coming from green1/GTLP1 to the right optical path. The p polarization is lost. After the last lens, there is a very fast switchable retarder, namely a ferro-electric liquid crystal (FLC) ${ }^{9}$ retarder. This is an optical component with two states which depend on the applied electrical field. In one state (off state) it acts as a normal window and in the other state (on state) as a half-wave plate changing the polarization state of the incoming light. During the pulsing of "green1", the FLC is set in the 'off state' and thus the polarization will be unchanged. The pre-polarizer after the FLC filters out the unwanted (p) polarization. PBS2 will reflect the rays to the 'LCOS 1' which will modulate according to the green information. Between the PBS and the light valve a quarter-wave plate (not shown in figure 2) will resolve the problem of skew ray depolarization ${ }^{10}$. After the PBS2, the analyzer will again filter out the unwanted (s) polarization. The combination of the pre-polarizer, the analyzer and the quarter-wave plate will increase the contrast dramatically. Subsequently LED "green2" will be pulsed and PBS1 transmits the p-polarization towards the lenses and the s-polarization will be again lost. The FLC is now in the 'on state' and changes the propagated light flux to s-polarization. After this, the propagation continues in the same manner. LCOS1 modulates again the same information. During one frame time, both green LEDs will have been pulsed once alternately.

\subsection{Red/Blue channel}

The second light valve is used to modulate the blue and red information alternately. As in the green channel, the LEDs are pulsed alternately at a higher current to get higher peak brightness. As shown in figure 3, the red and blue paths are combined, after the light pipes and the first lenses by a dichroic mirror.

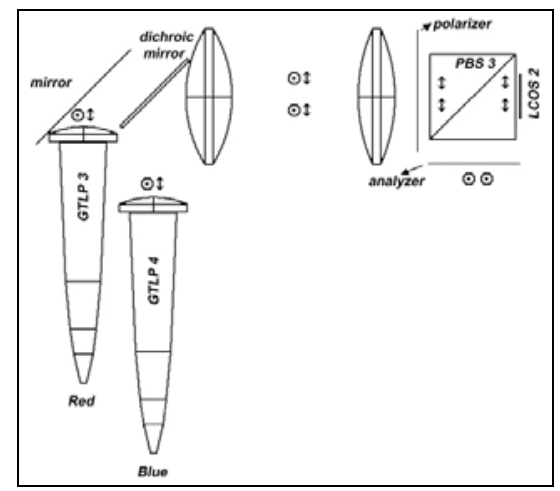

Figure 3: Red/blue channel

Up to the pre-polarizer, both polarizations are present in this part. The pre-polarizer filters out the (unwanted) spolarization. PBS 3 passes the flux that is modulated by LCOS2. We again added a quarter-wave plate between the PBS 
and the light valve to increase the contrast. After the PBS, the unwanted polarization (not modulated flux p-polarization) is filtered out by the analyzer. LCOS2 alternately modulates the red and blue information per frame.

\subsection{Complete architecture}

In the previous sections, the green and red/blue channels are described individually. In the total architecture (figure 1), both channels are combined by a large PBS. After the analyzer, the modulated flux in the green channel is in the ppolarization state and in the red/blue channel, it is in the s-polarization state, so we can combine them easily using a PBS. In addition, the PBS acts as an additional analyzer, increasing the contrast further. After the combining PBS, there is still a projection lens (not shown in figure 1).

With this architecture, using two light valves, we obtain a total light output similar to a three-light-valve approach because of the strong limitation of green LEDs. (Indeed in a three-panel system, we should drive the red and the blue LEDs at half power to obtain balanced colors). This makes our architecture more compact and less expensive. Compared with a single-panel color sequential architecture, we have a higher light output and reduced color breakup. Green is the most important color for projectors and by reserving one LCOS only for the green color we expect that the visibility of color breakup in our two-LCOS architecture will be suppressed considerably.

In our setup, the optical paths of the two channels (green and red/blue) are completely separated from each other. This leads to a high-quality projector with pure color images because cross talk is avoided between the green color, which contains most information, and the other colors.

\section{SIMULATIONS}

\subsection{Optical throughput efficiency, uniformity and contrast}

Computer simulation of this projection system was performed using ZEMAX to evaluate the optical throughput efficiency, uniformity and contrast. We have already described the system parameters in the second paragraph. We made here some more assumptions. The reflected flux from LCOS panels is $65 \%(90 \%$ fill factor, $4 \%$ absorption by the liquid crystal and $75 \%$ reflection coefficient), the efficiency of the projection lens is $80 \%$, the lenses and PBS are AR coated. By using the PBS parameters $R_{s}, T_{p}$ (table 2), its throughput can be calculated easily. For the red, green 1, green 2 and blue channels the resulting throughput is $\left(T_{p} R_{s} R_{s}\right),\left(R_{s} R_{s} T_{p} T_{p}\right),\left(T_{p} R_{s} T_{p} T_{p}\right)$ and $\left(T_{p} R_{s} R_{s}\right)$ respectively. The efficiency of the polarizer/analyzer is $97.6 \%$.

We performed the simulation with these assumptions and we obtained different results for the different channels as expected (difference in losses). Table 1 summarizes the results. The item 'other losses' contains the geometric losses and the polarization losses. Étendue efficiency is the ratio between the system étendue and the source étendue.

\begin{tabular}{|c|c|c|c|c|c|}
\hline & red & green 1 & green 2 & blue \\
\hline \multicolumn{2}{|c|}{ étendue efficiency [\%] } & 61.9 & 61.9 & 61.9 & 61.9 \\
\hline \multicolumn{2}{|c|}{ GTLP efficiency [\%] } & 81.1 & 81.1 & 81.1 & 81.1 \\
\hline \multicolumn{2}{|c|}{ LCOS efficiency [\%] } & 65 & 65 & 65 & 65 \\
\hline \multicolumn{2}{|c|}{ projection lens [\%] } & 80 & 80 & 80 & 80 \\
\hline \multicolumn{2}{|c|}{ PBS throughput [\%] } & 70.3 & 83.6 & 78.1 & 86.3 \\
\hline \multicolumn{2}{|c|}{ polarizer/analyzer $(2 \mathrm{x})[\%]$} & 97.6 & 97.6 & 97.6 & 97.6 \\
\hline \multicolumn{2}{|c|}{ Other losses [\%] } & 45.6 & 49.1 & 50.1 & 45.7 \\
\hline \multicolumn{2}{|c|}{ Total [\%] } & 7.97 & 10.21 & 9.73 & 9.81 \\
\hline \multicolumn{2}{|c|}{ On/Off contrast } & $>2000: 1$ & $>8000: 1$ & $>8500: 1$ & $>3000: 1$ \\
\hline \multirow{2}{*}{$\begin{array}{c}\text { ANSI } \\
\text { uniformity }\end{array}$} & brightest [\%] & +4.6 & +4.9 & +7.0 & +5.5 \\
\hline & dimmest [\%] & -6.9 & -8.2 & -7.9 & -5.7 \\
\hline
\end{tabular}

Table 1: Optical throughput efficiency, contrast and uniformity simulation results 
As expected, the optical throughputs are slightly different. The lower throughput of the red color can be explained by the lower performance of the PBS for this wavelength. The PBS performances for a light beam with $\mathrm{f}^{\#} 2.88$ are given in table 2. We also simulated contrast. For this, after the projection lens we measured the optical throughput in the on and the off state of the LCOS and calculated the on/off contrast by dividing these throughputs. The results are also presented in table 1 for each color. Red has the worst contrast, but this is normal because of the high leakage of the PBS for that color.

\begin{tabular}{|c|c|c|c|c|}
\hline & \multicolumn{2}{|c|}{ Simulated } & \multicolumn{2}{c|}{ HELF $^{\text {TM }}$} \\
\hline & $\mathrm{R}_{\mathrm{s}}[\%]$ & $\mathrm{T}_{\mathrm{p}}[\%]$ & $\mathrm{R}_{\mathrm{s}}[\%]$ & $\mathrm{T}_{\mathrm{p}}[\%]$ \\
\hline red & 85.00 & 97.25 & 99.85 & 80.00 \\
\hline green & 98.92 & 92.42 & 99.88 & 78.50 \\
\hline blue & 99.71 & 86.84 & 99.90 & 67.00 \\
\hline \multicolumn{3}{|c}{ Table 2: PBS performances for $\mathrm{f}^{\#} 2.88$}
\end{tabular}

Another important characteristic of the projector is the uniformity of the light beam that illuminates the LCOS panel. This is reflected in the uniformity of the image on the screen. The GTLP provides a uniform beam that is imaged to the LCOS. Figure 4 shows the flux distribution on the LCOS panels. We can observe that the brightness is uniform over the complete rectangle (light valve). The grid used in the simulation was 52 × 39 and we simulated 5 million rays. To confirm this, we calculated the uniformity numerically using the ANSI 13-point-measuring-grid method ${ }^{11}$. This method implies dividing the display into nine identical rectangles, measuring the illuminance at the center of each rectangle and calculating the average of these illuminances. Afterward, the illuminance in the four corner points is measured at $10 \%$ of the distance from the corner itself to the center of the display.

a)

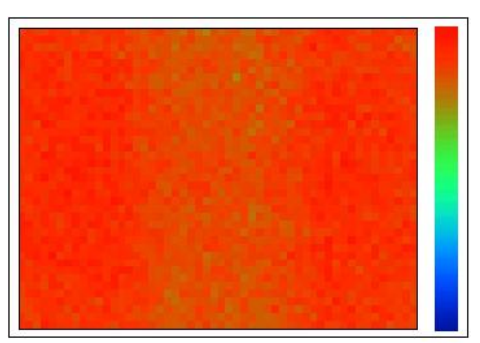

b)

c)

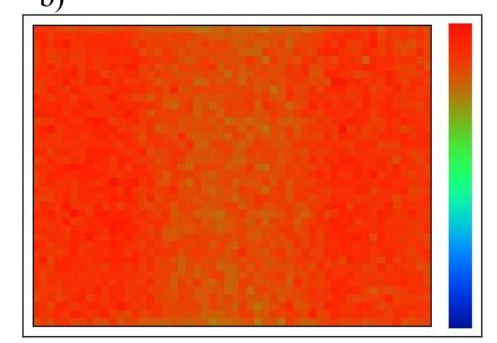

d)
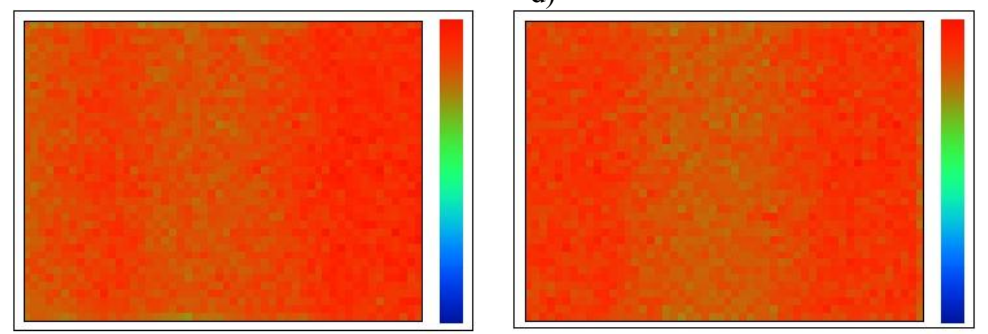

Figure 4: Illuminance distribution at the LCOS: a) green 1, b) green 2, c) red, d) blue

By comparing the brightest and the dimmest measurement location of these 13 points with the calculated average illuminance, we can express the uniformity numerically. We did this for the four images shown in figure 4 and the results are summarized in table 1 . These results confirm the uniformity seen in figure 4 . We can conclude that we have a very good uniformity.

\subsection{LED performances and color balancing}

The LED is radiating directly in the air and thus the extraction efficiency is not very high. By coupling the LEDs optically to our light pipes (GTLP) as described in the previous sections, the source étendue will be 2.3 times higher 
$\left(\mathrm{n}^{2}\right)$. In our simulation, the increase of the étendue is already taken into account. Not only does the étendue increase, but also the light extraction from the LEDs. Theoretically the maximal achievable gain would be $2.35^{12}$. The actual gain is usually not sufficient to maintain the brightness (lumen/étendue) and is a little bit lower, namely about 2.

In this architecture, we pulse red/blue and green $1 /$ green 2 alternately with $50 \%$ duty cycle at a current twice as the nominal current which is $1 \mathrm{~A}$. However the peak current for red LED will be limited at 1.4 A. Higher currents will decrease the light output. The pulse efficiency, which is defined as the peak flux divided by two times (in case of $50 \%$ duty cycle) the average flux, should be $100 \%$ in the ideal case but because of non-idealities of the LEDs (increasing voltage and decreasing efficiency by increasing current), the efficiency is limited to respectively $67.5 \%, 69.9 \%$ and $77.3 \%$ for red, green and blue LED. Table 3 gives a summary of lumen outputs of the LEDs adjusted with optical coupling and pulsing efficiencies.

\begin{tabular}{|c|c|c|c|c|}
\hline & red & green 1 & green 2 & blue \\
\hline lumen output [lm] & 321 & 321 & 321 & 83 \\
\hline optically coupling & 2 & 2 & 2 & 2 \\
\hline Pulsing efficiency & 0.675 & 0.699 & 0.699 & 0.773 \\
\hline Total [lm] & 433 & 449 & 449 & 128 \\
\hline $\begin{array}{c}\text { lumen fraction for D65 } \\
{[\%] \text { at the input }}\end{array}$ & 34.55 & 30.11 & 31.60 & 3.74 \\
\hline
\end{tabular}

Table 3: Summary of lumen outputs of LEDs with applied gains and balancing fractions

Based on the color coordinates of the LEDs, we can calculate the lumen fractions needed for a balanced white color (D65). We measured the color coordinates with a spectrometer and performed the calculations. For a balanced white (D65) color, we require $66.34 \%$ green (33.17\% green 1 and $33.17 \%$ green 2 ), $29.71 \%$ red and $3.96 \%$ blue light from that type LEDs expressed in lumens. That is the ratio of colors in the projected flux. At the source, the ratios will be different because of different throughput efficiencies (see table 1). If we calculate back to the source the lumen ratios should be $34.55 \%$ red, $30.11 \%$ green $1,31.60 \%$ green 2 and 3.74\% blue to have D65 white on the screen. We also have different ratios for the green LEDs to obtain an equal lumen output on the screen per green LED, otherwise we might observe flicker (intensity differences). The results are given in table 3.

\subsection{Results}

Now we can combine the results from the previous paragraphs to obtain the final results. By using the data from table 1 and table 3 we calculated the actual output of this two-LCOS projector. The results are given in table 4 . We have a balanced output of $119 \mathrm{~lm}$ with a total optical throughput of $9.3 \%$.

\begin{tabular}{|c|c|c|c|c|}
\hline & red & green 1 & green 2 & blue \\
\hline input [1m] & 443 & 386.1 & 405.1 & 48 \\
\hline throughput efficiency [\%] & 7.97 & 10.21 & 9.73 & 9.81 \\
\hline projected flux [1m] & 35.3 & 39.42 & 39.42 & 4.71 \\
\hline projected flux [1m] & 35.3 & 78.84 & 4.71 \\
\hline lumen ratios [\%] & 29.71 & \multicolumn{5}{|c|}{66.34} & 3.96 \\
\hline Balanced white D65 [lm] & $\mathbf{1 1 8 . 8 5}$ \\
\hline
\end{tabular}

Normally green is the limiting color in LED projectors, but in this architecture and with these LEDs the red LED is the limiting color and is driven at maximum power, the green LEDs almost at maximum power. The blue LED has the remainder of optical power $(62 \%)$.

Table 1 presented the individual contrasts of each channel, but we are also interested in the on/off contrast of the total projector in a color balanced situation. Therefore, we must calculate the flux in the off state, with the right lumen fractions at the input. The ratio of the balanced white lumens to this off state flux is the total contrast and amounts about 2500:1. 
We simulated a relatively compact and efficient LED projector with two LCOS light valves. The projector produces 119 Im D65 light flux with a high contrast ratio 2500:1 and a very good uniformity, within a system étendue lower than 20 $\mathrm{mm}^{2} \mathrm{sr}$. These results are promising and we decided to build an experimental setup of this design.

\section{DEMONSTRATOR SETUP AND EXPERIMENTAL RESULTS}

In this paragraph we describe the experimental setup of the designed two-LCOS projector and discuss the results. We begin the discussion by analyzing the differences between the simulated model and the experimental setup. We give the throughput efficiencies of the several channels and make a flux balance of the LEDs. After that the electronic circuits are explained briefly. Next, the performances of the demonstrator are measured and the results are discussed. Finally we offer some suggestions to improve the light output in the future.

\subsection{Experimental setup}

We built the architecture given in figure 1. Because of the mechanical restrictions (size holders) we omitted the cold mirrors and placed the GTLP1-GTLP2 and GTLP3-GTLP4 orthogonally. That will extend the architecture, but in this stage that is not so crucial. We built here a principle demonstrator and in a real product the overhead volume can be minimized. A photograph of this setup is shown in figure 5. We covered the setup as much as possible with black cardboard to avoid that the scattering light decreases the contrast. The setup is built on a black painted sheet and the holders are also painted black.

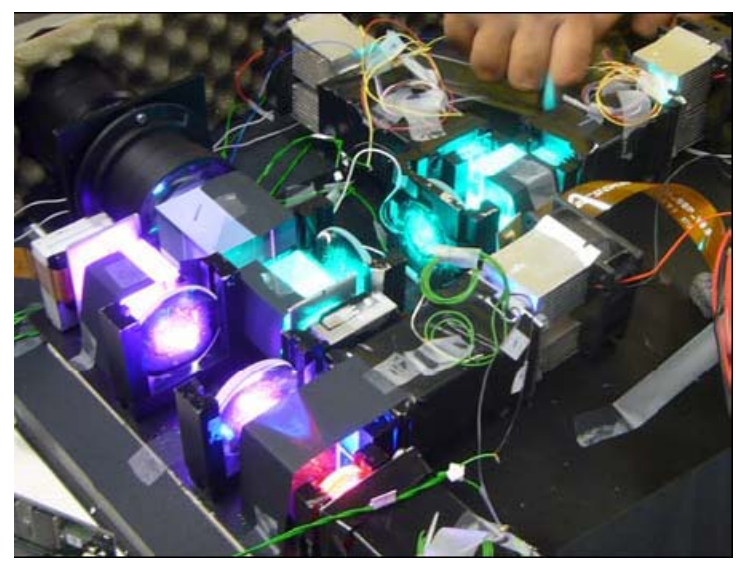

Figure 5: Two-LCOS demonstrator

Most optical components are AR-coated as assumed, but not all of them. That will introduce extra Fresnel losses. The exit surface of the GTLPs is not coated. Only one surface (entering surface of the red/blue light) of (the large) PBS4 is coated. The dichroic mirror between the red and the blue channels is also not coated. That will mainly influence the red light flux. The polarizers, the analyzers, $\lambda / 4$-waveplates and the LCOS panels are also not AR-coated. The light will meet 6 uncoated glass-air crossings between the PBS and the micro display. We minimized it to two crossings by coupling the $\lambda / 4$-waveplates and the LCOS panels optically with index matching gel.

In the previous section (table 1) we saw that the simulation results of the designed GTLP were promising. We fabricated $^{7}$ these components in quadruple (one per LED) and measured the performances experimentally. The fabricated GTLPs have 2 to $9 \%$ lower efficiency. The pipe material is Plexiglas $\left(\mathrm{n}_{\text {Plexiglas }}<\mathrm{n}_{\mathrm{BK} 7}\right)$ and the source étendue is thus lower than simulated. That means that the étendue efficiency will be higher $(64.34 \%)$. 
The FLC is, unlike the simulated architecture, placed after the second lens. The clear aperture of the FLC is limited and after the third lens it stops some of the light rays. Besides after the second lens (focal plane of the first lens) it acts as a diaphragm that stops the light rays with an angle above $11.3^{\circ}$. The maximum allowed angle in this system is $10^{\circ}$. This component introduces a loss ${ }^{1}$ of about $7.8 \%$. The polarizer and analyzer were represented as Jones-Matrices in the simulation. That is an idealized representation with a higher efficiency than in the real case. In the setup we used Proflux High Contrast polarizers from Moxtek ${ }^{13}$. These polarizers have a measured throughput of respectively $87.2 \%$, $88.85 \%$ and $83.7 \%$ for red, green and blue light with $\mathrm{f}^{\#} 2.88$.

The PBS that we used in this setup is the $\mathrm{HELF}^{\mathrm{TM}}{ }^{8}$ PBS from Oerlikon. The parameters of this component for a light beam with $\mathrm{f}^{\#} 2.88$ are given in table 2 . Compared with the simulated model the transmission of p-polarized light is lower. That will lower the throughput and the contrast. The throughput can be calculated for each channel by using its $R_{s}$ and $T_{p}$ values. The throughput for the red, green 1, green 2 and blue channels are respectively $\left(T_{p} R_{s} R_{s}\right),\left(R_{s} R_{s} T_{p} T_{p}\right)$, $\left(T_{p} R_{s} T_{p} T_{p}\right)$ and $\left(T_{p} R_{s} R_{s}\right)$. The results are given in table 5 .

The throughput of the LCOS panels in the simulation model is assumed to be $65 \%$ ( $90 \%$ fill factor, $4 \%$ absorption by the liquid crystal and 75\% reflection coefficient). In the setup, we used QXGA LCOS panels from Gemidis. These panels have a better reflection $(80 \%)$, but they are not AR-coated. That causes an extra Fresnel loss of $2 \times 4 \%$. The effective throughput will be $63.7 \%$.

$80 \%$ throughput (assumed value) is a reasonable value for a projection lens. However the only projection lens available to us with a sufficient working distance, was a zoom lens with an $\mathrm{f}^{\#}$ between 3.0 and 4.0. Because of the f-number limitation the lens will stop a part of the rays. Besides, it is a dark system because of the presence of several lenses. In the state that we use it, the transmission is respectively $63 \%, 57.2 \%$ and $56.3 \%$ for red, green and blue light (instead of $80 \%$ ). The projection screen has a measured reflectivity of $92.7 \%, 92 \%$ and $92.7 \%$ for $\mathrm{R}, \mathrm{G}, \mathrm{B}$ colors. In the simulation we did not take that into account.

In table 5 we have summarized these results. The item 'other losses' contains not only the geometrical losses and the polarization losses, but also the losses due to the FLC and the dichroic mirror. The last row gives a comparison between the experimental and the simulated throughput. The total efficiency of the architecture is lower than expected, but all the losses are localized. The decrease of the efficiency is lowest for the red channel $(60 \%)$ and is large for the other channels (+/- 30\%). It means that the red channel will probably not be the limiting color.

\begin{tabular}{|c|c|c|c|c|}
\hline & red & green 1 & green 2 & blue \\
\hline étendue efficiency [\%] & 64.34 & 64.34 & 64.34 & 64.34 \\
\hline GTLP efficiency [\%] & 79.65 & 76.34 & 76.86 & 73.73 \\
\hline LCOS efficiency [\%] & 63.7 & 63.7 & 63.7 & 63.7 \\
\hline projection lens [\%] & 63 & 57.2 & 57.2 & 56.3 \\
\hline projection screen [\%] & 92.7 & 92 & 92 & 92.7 \\
\hline PBS throughput [\%] & 79.76 & 61.5 & 48.32 & 66.9 \\
\hline polarizer/analyzer (2x) [\%] & 87.2 & 88.85 & 88.85 & 83.7 \\
\hline other losses [\%] & 41.05 & 41.44 & 42.50 & 43.72 \\
\hline Total [\%] & $\mathbf{4 . 7 5}$ & $\mathbf{3 . 3 1}$ & $\mathbf{2 . 6 9}$ & 3.23 \\
\hline fraction exp/sim & 0.60 & 0.32 & 0.28 & 0.33 \\
\hline
\end{tabular}

Table 5: Optical throughput efficiency measurement results

Before we discuss the measured light output of the LEDs, we will explain briefly the driving principle of the QXGA LCOS panels, because this is necessary to understand the chosen duty cycles for red and blue LEDs. The video is delivered at a frame rate of $60 \mathrm{fps}$. However the panels work at $120 \mathrm{~Hz}$ which means that the panels are written twice (line per line) with the same information during one frame period. That is very important for the red/blue channel because of the color-sequential operation. During the first time, when a frame is written, the panel will contain mixed information of both color components and thus cannot be illuminated by an LED. During the second write cycle, the panel will have only information of one component and can be illuminated with LED of that color. This means that the duty cycle for the red and the blue LEDs should not exceed $25 \%$; otherwise the information will be illuminated with the wrong color on a part of the display. In that case, color crosstalk between the red and the blue channel will be 
unavoidable. Normally, the duty cycle needs to be even shorter because the liquid crystal has a certain response time ( $2.3 \mathrm{~ms}$ for the Gemidis panels at room temperature). We have experimentally verified that duty cycle of $25 \%$ is not disturbing and the color crosstalk is negligible. Now we will pulse the red and blue LEDs with $25 \%$ duty cycle (DC) instead of $50 \%$. The time diagram of the LCOS illumination is shown in figure 6.

The light flux of the OSTAR LEDs is given in the datasheets ${ }^{6}$. These values can be slightly different for several samples. We measured the samples that we used in the setup. The flux is respectively $319 \mathrm{~lm}, 319 \mathrm{~lm}, 332 \mathrm{~lm}$ and 66.2 $1 \mathrm{~m}$ for the red, green1, green2 and blue LEDs. Compared with the values in the datasheets (table 3), mainly the blue LED has a lower optical output. Next, we coupled them optically with the light pipes. The enhancement is [83.4\%, $76.4 \%, 87.7 \%, 19.0 \%$ ] for the (R, G1, G2, B) LEDs. The enhancement of the blue channel is inferior. The main reason is the not-ideal flatness of the coupling surface of that GTLP. The pulsing efficiency of the green LEDs remains unchanged $\left(\mathrm{DC}=50 \%\right.$ and $\left.\mathrm{I}_{\text {peak }}=2 \mathrm{~A}\right)$, the red LED decreases to $0.399\left(\mathrm{DC}=25 \%\right.$ and $\left.\mathrm{I}_{\text {peak }}=1.8 \mathrm{~A}\right)$ and the blue LED decreases to $0.458\left(\mathrm{DC}=25 \%\right.$ and $\left.\mathrm{I}_{\text {peak }}=2 \mathrm{~A}\right)$. Now we can calculate the total light output of each channel. It is given in table 6. In the last row again a comparison is given between the experimental and the simulated light flux. The flux of the green LEDs is nearly the same as assumed. The red and blue LEDs are harmed by the color-sequential operation (duty cycle $=25 \%$ ). Moreover, the blue LED is weaker as expected and has a bad optical coupling.

\begin{tabular}{|c|c|c|c|c|}
\hline & red & green 1 & green 2 & blue \\
\hline lumen output [1m] & 319 & 319 & 332 & 66.2 \\
\hline optically coupling & 1.834 & 1.764 & 1.877 & 1.19 \\
\hline pulsing efficiency & 0.399 & 0.699 & 0.699 & 0.458 \\
\hline Total [lm] & 233.4 & 393.4 & 435.5 & 36.1 \\
\hline fraction exp/sim & 0.54 & 0.88 & 0.97 & 0.28 \\
\hline
\end{tabular}

Table 6: Summary of measured lumen outputs of LEDs with applied gains

If we combine table 5 and 6 (with lumen fractions of D65 in mind), we can conclude that blue will be the limiting color and the other LEDs will have the remainder of optical power. To increase the light output of the projector and make optimal use of the available LED power, we need more blue light. We can realize that by increasing the duty cycle and permitting some blue color crosstalk in the red information. Blue light in the red channel is not very disturbing. We experimentally verified that, the gain of light output outweighs the small effect of blue crosstalk. We chose a $35.9 \%$ duty cycle for the blue LED and $\mathrm{I}_{\text {peak }}=2 \mathrm{~A}$. The pulsing efficiency will be $57.8 \%$ and the total blue flux $45.7 \mathrm{~lm}(+27 \%)$. The red and green LEDs are now in balance (D65) and will have no remainder anymore.

\subsection{Driving electronics}

In this section we will briefly describe the driving circuits of the projector. Most of them are well-known circuits in the electronics. We will not discuss them in detail, but will explain their functions in the total system. The Gemidis light valves are delivered with their electronic drivers that consist of one 'Balder' and two 'Modi' boards (one per panel). The balder board is connected with the image source (e.g. a personal computer) and is getting the video stream at $60 \mathrm{fps}$ but internally doubles this to $120 \mathrm{fps}$. A synchronization pulse will be generated per $60 \mathrm{~Hz}$. In this board the frame information will be converted into R, G, and B components presented by 10-bit values. Each of these components will normally be sent to a modi board that is connected with an LCOS panel. The modi boards will calculate the necessary electric fields and will apply them to the panels to reconstruct an image. In our two-panel system, we have an adapted balder board that alternately swaps the blue and the red output channel. The green channel will get two times green information while the red (blue) channel will get alternately red (blue) and blue (red) information. By using one modi board (and one panel) that is connected with the red or the blue channel of the balder board, both color informations will be modulated alternately. The real frame rate will be $30 \mathrm{fps}$. The time diagram of this system is given in figure 7 . Here is the 'write' $=$ 'illumination' $=(1 / 120) \mathrm{s}$ and 'total frame time' $=(1 / 30) \mathrm{s}$ 


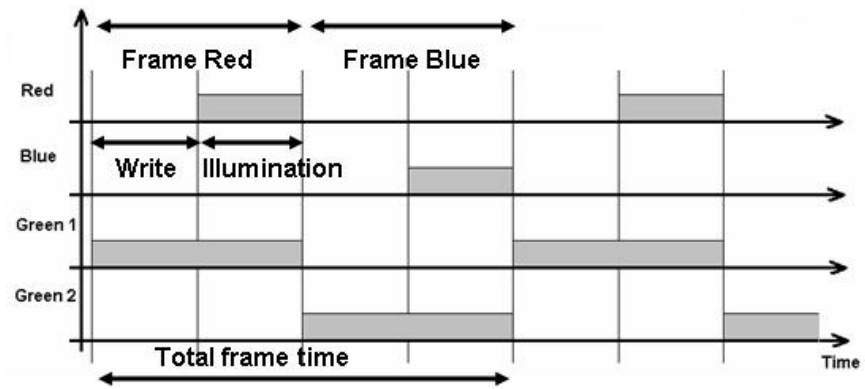

Figure 6: Time diagram LCOS illumination

The synchronization pulse will be used to switch the LEDs at the right moment. This pulse is not generated at the beginning of the frame and needs to be delayed. Therefore we built a timer (555) circuit in monostable operation mode, where the delay time is adjustable by a variable resistor. Besides, we need 5 transistor circuits to drive the LEDs and the FLC. The current through these circuits will be adjusted by a current source. Green LEDs have a fixed duty cycle of $50 \%$. Red and blue LEDs have an adjustable duty cycle that is realized with the help of analog-to-digital-converters (ADCs). All these circuits are connected with a microcontroller. The microcontroller is the heart of the driving electronics. As input it uses the delayed sync pulse (interrupt input) and the 8-bit values of the ADCs. From these values the controller can calculate the duty cycle for red and blue LEDs. Each time a delayed sync triggers the controller, it will drive two of the four LED circuits (G1+R or G2+B) plus the FLC circuit. The next pulse will drive the other two LED circuits. Green LEDs will alternate each other (50\% duty cycle) and red and blue LED will be electronically switched off after the adjusted duty cycle. The block diagram of the circuits is given in figure 7 . By using the sync pulse from the balder board, the illumination will be synchronized with the modulation (LCOS panels).

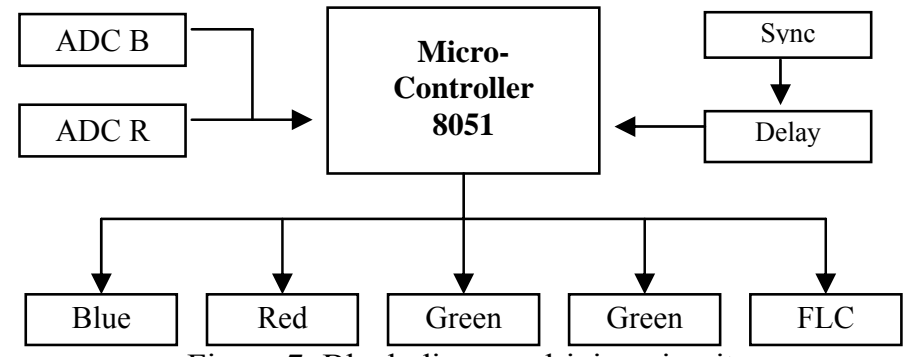

Figure 7: Block diagram driving circuit

\subsection{Results}

Based on the experience from section 4.1, we drive the LEDs with respectively $25 \%, 50 \%, 50 \%$ and $35.9 \%$ duty cycle (R, G1, G2, and B) to have a balanced white point (D65) and measure the light output, uniformity and contrast on the projection screen. The uniformity is measured according to the ANSI 13-point-measuring-grid method ${ }^{11}$. We measured two types of contrast: the on/off-contrast and the ANSI-contrast. The on/off contrast is evident and is done by measuring the brightness of a white $(100 \%)$ and a black image and taking the ratio. On the contrary, the ANSI contrast ${ }^{11}$ is somewhat complicated. We project a chessboard image and measure the brightness of the center of black and white rectangles. The average luminance value of the bright rectangles is divided by the average luminance value of the dark rectangles. The ANSI contrast is the ratio between these values. The results of these measurements are summarized in table 7. To measure the channels individually, we switched only the measuring channel (LED) on.

We can observe that the experimental results are lower than the simulation results. The reason is already discussed in section 4.1. Nevertheless, the projector has a moderate light output with good uniformity and reasonable contrast. While the uniformity is measured on the light valve in the simulation, we measured it on the screen in the experimental setup. The decrease of the uniformity is probably due to the projection lens and the fabricated GTLP. The lower contrast ratio has several reasons. In the simulation, the components like LCOS panels, $\lambda / 4$-waveplate and polarizer/analyzer are 
idealized; in practice they are limiting the contrast. The simulated PBS has a better intrinsic contrast and all the components in the simulation are AR-coated.

\begin{tabular}{|c|c|c|c|c|c|c|}
\hline \multicolumn{2}{|c|}{} & red & green 1 & green 2 & blue & D65 \\
\hline \multicolumn{2}{|c|}{ input [1m] } & 233.4 & 393.4 & 435.5 & 45.7 & \\
\cline { 1 - 6 } throughput efficiency [\%] & 4.75 & 3.31 & 2.69 & 3.23 & \\
\hline \multicolumn{2}{|c|}{ projected flux [lm] } & 11.09 & \multicolumn{2}{|c|}{24.74} & 1.48 & $\mathbf{3 7 . 3 1}$ \\
\hline \multirow{2}{*}{$\begin{array}{c}\text { ANSI } \\
\text { uniformity }\end{array}$} & brightest [\%] & +8.6 & \multicolumn{2}{|c|}{+6.5} & +12.6 & $+\mathbf{6 . 3}$ \\
\cline { 2 - 6 } & dimmest [\%] & -4.5 & -14.0 & -8.3 & $\mathbf{- 1 0 . 1}$ \\
\hline \multirow{2}{*}{ contrast } & On/Off & $512: 1$ & $462: 1$ & $350: 1$ & $\mathbf{4 7 7 : 1}$ \\
\cline { 2 - 6 } & ANSI & $57.2: 1$ & $61.9: 1$ & $37: 1$ & $\mathbf{6 1 . 6 : 1}$ \\
\hline
\end{tabular}

Table 7: Experimental performances of the 2-LCOS projector

By using LEDs as light source, the projector gets a larger color gamut that will result in a better color quality and vivid colors. Compared with a classic television system like NTSC, this LED projector produces $65 \%$ more colors. These colors will also be more saturated. Thanks to the semi-color-sequential operation, one panel is permanently reserved for the green color (most important color), the color breakup and crosstalk are strongly reduced. We still have some flicker because of the low frame rate (30 fps). The flicker is most pronounced in the red light. Green light is permanently present and will not cause any flicker and the intensity of the blue light is too minor to notice any flicker. In figure 8 , we show a picture of the projected image. In the picture, a number of objects with their natural color are imaged. The details are clearly visible and the contrast is good. The colors are truthful and very bright.

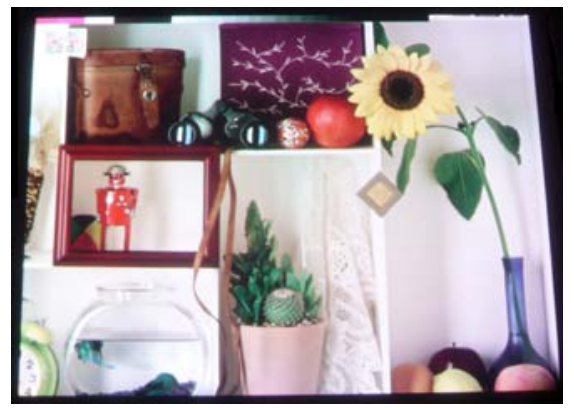

Figure 8: Picture of projected image

\subsection{Future perspectives}

Compared with the simulation results, the demonstrator performance is too low (31\%), but this can be still improved considerably. We will distinguish here two kinds of improvements: a short term improvements and a long term improvements. The short term improvements can in principle be applied immediately. These are adding anti reflective coatings on all optical surfaces (higher throughput and contrast), using a high efficiency projection lens ( $>80 \%)$ with the right f-number, an automated fabrication process (instead of manual) for the GTLP production to avoid any defects, using LEDs samples with a high brightness group ${ }^{6}$ (binning) and using PBSes that have better performances (higher $\mathrm{T}_{\mathrm{p}}$ ) such as the simulated PBS.

The long term improvements are today impossible to apply and need some technological advances. These improvements are concerning the LED- and the LCOS-technology. The luminance of the LEDs is still increasing, ${ }^{4,14}$ and they will be more powerful in the future. Besides, there is also ongoing research about polarized LEDs ${ }^{15}$ which will be very suitable for LCOS projectors. That will increase the brightness and the wall plug efficiency strongly. On the side of the LCOS panel, there are also some developments. In the current generation of LCOS panels, the frame information cannot be written immediately onto the whole panel which is limiting the available illumination duration in color sequential systems. However there is ongoing research towards smart pixel LCOS panel architectures ${ }^{16}$ that is resolving this problem. By using an underlying frame memory, the frame information can be immediately written to the LCOS panels. 
In our architecture, we could then pulse the red and blue LEDs with $50 \%$ duty cycle instead of $25 \%$, which would effectively double the light output. We can also increase the frame rate to avoid flicker. By adding these improvements (short and long term) the light output of the projector will be highly increased.

\section{CONCLUSIONS}

We presented a relatively compact and efficient LED projector with two LCOS light valves. One light valve is alternately modulating the red and the blue information; while the other is permanently modulating the green information to get a balanced white color. The projector has a good image quality and a moderate light output. By using two panels instead of three, we have achieved an optimal cost-performance/quality ratio.

We first simulated the projector that produces $119 \mathrm{~lm}$ D65 light output with a high contrast ratio 2500:1 and a very good uniformity, within a system étendue lower than $20 \mathrm{~mm}^{2} \mathrm{sr}$. After that we built a demonstrator setup of this 2-LCOS projector and characterized it experimentally. We also designed custom electronics to drive the LEDs synchronously with the LCOS panels. The measured light output is $37.3 \mathrm{~lm}$ D65 color point. The on/off contrast is 477:1 and the ANSI contrast is $61.6: 1$. The uniformity is still very good. The differences between the simulation and experimental results are mainly due to the used components and they are discussed thoroughly in this paper. The use of LEDs results in a larger color gamut which means that the colors seem to be more truthful and vivid. In spite of the semi-color-sequential operation, the color breakup and crosstalk are limited to acceptable values. The main remaining problem is that of flicker in the red and the blue color because of the limited frame rate $(30 \mathrm{fps})$. We are convinced that the remaining issues will be solved by future improvements in LED and LCOS technology.

\section{ACKNOWLEDGMENTS}

This work was partially funded by the 'Instituut voor de aanmoediging van innovatie door Wetenschap en Technologie in Vlaanderen' (project name is “Compact high-quality LED projection systems”).

\section{REFERENCES}

1. H. Murat, H. De Smet, D. Cuypers, Y. Meuret, H. Thienpont, M. Vervaeke, L. Desmet, "Increased lumens per étendue by combining pulsed light-emitting diodes", Optical Engineering, Vol 45, n 3, page 034002, March 2006.

2. G. Harber, M. Keuper, S. Paolini, "Performance of High Power LED Illuminators in Color Sequential Projection Displays", Proceedings of the 10 $0^{\text {th }}$ International Display Workshop, IDW '03, pp1585-1588, Fukuoka, 2003.

3. G. Harber, S. Paolini, M. Keuper, "Performance of high-power LED Illuminators in Projection Displays", SID Microdisplay 2002 Digest of Papers, Westminster, CO (2002).

4. R. V. Steel, "The story of a new light source", Nature Photonics, Vol 1, pp 25-26, 2006.

5. M. H. Keuper, G. Harber, S. Paolini, "RGB LED Illuminator for Pocket-Sized Projectors”, SID’04 Digest of Technical Papers, pp. 943-945, 2004

6. http://catalog.osram-os.com

7. H. Murat, A. Gielen, H. De Smet, "Gradually tapered light pipes for illumination of LED projectors", Journal of SID, in progress, 2007.

8. http://www.oerlikon.com/ecomaXL/index.php?site=OPTICS EN helf pbs

9. V. Presnyakov, Z. Lui, V.G. Chigrinov,"Fast optical retarder using deformed-helical ferroelectric liquid crystals", Proceedings of the SPIE, Vol 5970, pp. 426-435, 2005

10. A.E. Rosenbluth, M. Lu, K.-H. Yang, K. Ho, R.N. Singh, T. Nakasogi, "Correction of contrast in projection systems by means of phase-controlled prism coatings and band-shifted twist compensators", Projection Displays 2000: Sixth in a Series, Vol 3954, pages 63-90, San Jose, 2000. 
11. ANSI/PIMA IT7.227-1998: “American National Standard for Audiovisual Systems - Electronic Projection Variable resolution projectors", 1998

12. H. Zou, A. Schleicher, J. Dean, "Single-Panel LCOS Color Projector with LED Light Sources", SID 05 Digest of Technical Papers, pp. 1698-1701, 2005.

13. http://www.moxtek.com/optics/visible light.html

14. R. H. Haitz, Light source for projection display, US patent \#5,971,545 issued October 26, 1999.

15. J. Wheatley, C. Leatherdale, A. Ouderkirk, POLARIZED LED, EP20050801187, 3M Center, 2007-11-07.

16. H. De Smet, J. Van Den Steen, G. Van Doorselaer, A. Van Calster, Refresh pixel circuit for active matrix, EP 1516314 (A1), Gemidis NV, 2005-03-23. 